\title{
Dynamic phenomena for charged clusters in cryogenic liquids
}

\author{
I. Chikina \\ CEA Saclay, LIONS, IRAMIS, CEA, F-91191, Gif Sur Yvette, France \\ S. Nazin and V. Shikin \\ Institute of Solid State Physics of Russian Academy of Sciences, Chernogolovka 142432, Moscow dist., Russia \\ E-mail: shikinv@yandex.ru \\ Received December 1, 2010

\begin{abstract}
Discussed in the paper are resonance phenomena in electrolytes related to possible relative motion of the charged core and hydrate (solvate) shell of each cluster. The resonances are shown to contain important information on the internal structure of clusters. Special attention is paid to the process of formation of the cluster associated mass in the solvent.
\end{abstract}

PACS: 66.10.-x Diffusion and ionic conduction in liquids; 66.10.Ed Ionic conduction.

Keywords: diffusion and ionic conduction in liquids.

The problem of solvent inhomogeneity in the vicinity of point-like charges important for most effects involving various charged clusters is one of the tasks very poorly treated in the theory of electrolytes. Its quantum-chemical treatment (e.g., see Refs. 1 and 2) employing concepts of the first and next coordination spheres around the charged center and predicting complete or partial solvent crystallization there the hydration (solvation) effect only approximately accounts for the role of external environment in cluster formation. Current microscopic theories $[3,4]$ providing detailed description of the short-range order in the structure of a homogeneous liquid do not work within the local density enhancement $\delta \rho(r)$ around the ion. The existent experimental techniques allow as a rule to obtain only integral characteristics of the cluster (e.g., hydration (solvation) energy in the thermodynamics of electrolytes $[1,2])$ or lackluster data on effective masses of charged clusters derived from ultrasonic measurements $[5,6]$.

In these extremely adverse for development of appropriate theory conditions (strong interaction between the particles inside the polaron, substantial spatial inhomogeneity, lack of firmly established experimental data) it is natural to search for additional sources of information capable of elucidating the cluster structure. Considered in the present paper is one of the approaches in that direction which has not yet been discussed in the literature. The idea is to consider the resonances (which we call the structure resonances) arising in the course of relative motion of the core ion and the adjacent neutral shell of the charged cluster. It should be emphasized that, in our opinion, the very concept of structure resonances has already been dealt with in experiments on charged nanodroplets [7-10]. By employing the technique allowing to produce extremely small droplets (containing a few water molecules: $1,2,3 \ldots$ whose number is measured in mass-spectrometer experiments) and charging them with single protons, the authors of Refs. 7-10 observed excitation of droplets in external high-frequency field and calculated the observed resonance frequencies with the first-principles methods employing the molecular dynamics technique. Yielding the numbers which are consistent with available experimental data, this approach tells practically nothing on physics of observed phenomena. Under these conditions our purpose is not only transferring the ideas of Refs. 7-10 to clusters in liquid; here the problem is less promising in the sense of the sharpness of resonances, but it is more important in its essence (the problem formulation is more natural, the clusters need not be fabricated artificially since they are already available and have precisely the same size in each electrolyte; in addition, the resonance frequency can be the major characteristic allowing to identify the charged cluster) involving the discussion of physical nature of the resonances in nanodroplets. Further, the proposed quasimacroscopic approach allows one to trace how the macroscopic 
liquid characteristics (such as dielectric constant, surface tension, etc.) arise as the nanocluster size is increased. Similar problems are often considered in condensed matter physics (appearance of collective Fermi level or superconducting order parameter in small metal particles, development of superfluid properties in liquid nanodroplets, etc.)

1. Let us first show (the central statement of the present paper) that a single charged cluster can be resonantly excited in the following way (for simplicity, we consider the classical case). The solvated ion contains a charged nucleus of mass $m_{i}$ and adjacent density enhancement consisting according to Refs. 1 and 2 of at least 6-8 solvent molecules in the first coordination sphere firmly bound to the core charge due to the ion-dipole interaction. Let $M \gg m_{i}$ be the shell mass. The external electric field accelerates the mass $m_{i}$ carrying the electric charge. As to the neutral shell, it is only accelerated due to some elastic coupling to the core charged particle. Thus, dynamics of the entire complex $m_{i}+M$ is described by two equations

$$
-m_{i} \omega^{2} x=-k\left(x-x_{1}\right),-M \omega^{2} x_{1}=-k\left(x_{1}-x\right)
$$

where $k$ is the elastic interaction coefficient between the components. This quantity is assumed to be finite and is actually a new phenomenological parameter of the charged cluster we are interested in.

Finding from the second equation in (1) the displacement $x_{1}$

$$
x_{1}=\frac{k x}{k-M \omega^{2}}
$$

and substituting it into the first equation one easily obtains the proper dipole frequency of the cluster as a whole:

$$
k-m_{i} \omega^{2}=\frac{k^{2}}{k-M \omega^{2}}
$$

or

$$
\tilde{\omega}^{2}=\frac{1+\gamma}{\gamma}, \tilde{\omega}=\frac{\omega}{\sqrt{k / m_{i}}}, \gamma=M / m_{i} .
$$

The quantity $\tilde{\omega}^{2}$ varies from unity for $\gamma \gg 1$ up to 2 for $\gamma \simeq 1$, according to well-known properties of dynamics of two coupled particles. We use the term "dipole" for the frequency (3) since it can be excited with a uniform electric field.

In its pure form, the resonance (3) is only possible for single charged droplets and, in our opinion, it is exactly the case addressed in Refs. 7-10. However, if positive or negative clusters in liquid solvents are discussed, the problem described by Eq. (1) needs some refinement since in that case both the associated mass $M^{*}$ and effective viscosity of the environment should be taken into account. If the frequency range in the vicinity of the resonance (3) allows to speak of the well-formed quantity $M^{*}$, its contribution to equations of motion (1) is rather simple:

$$
M_{\mathrm{eff}}=M+M^{*}, M^{*}=\frac{2}{3} \pi R_{i}^{3} \rho_{l}, R_{i}>R_{0},
$$

where $M$ is again the shell bare mass, $R_{i}$ is effective external cluster radius in ideal hydrodynamics, $R_{0}$ is some internal cutoff radius for the structural singularities associated with properties of the ion-dipole interaction $V_{i d}$, and $\rho_{l}$ is the solvent mass density.

However, generally, the associated mass frequency dispersion becomes important in calculations of the structure resonances. This phenomenon (which is also of structural origin) can be taken into account by adding the hydrodynamic drag force $\mathbf{F}_{\text {hyd }}$ into the cluster equation of motion. Classical hydrodynamics yields two asymptotic expressions for the force $\mathbf{F}_{\text {hyd }}$ acting on a sphere of radius $R_{i}$ corresponding to its motion in either ideal or viscous (Stokes formula) liquids (e.g., see Ref. 11). In the first case, employing the frequency representation where all quantities are proportional to $\exp (-i \omega t)$ one has

$$
\mathbf{F}_{i d}(\omega)=2 M^{*} \omega \mathbf{v} \frac{\left[-q^{3} R_{i}^{3}+i\left(2+q^{2} R_{i}^{2}\right)\right]}{\left(4+q^{4} R_{i}^{4}\right)}, q=s / \omega,
$$

where $s$ is the sound velocity, $q$ is the wave number (the dispersion law is assumed to be linear), and $\mathbf{v}$ is the amplitude of the cluster velocity as a whole. In the limit $q^{2} R_{i}^{2} \ll 1$ the associated mass $M^{*}(\omega)$ defined as proportionality coefficient in the ratio

$$
\operatorname{Im} F_{i d}(\omega)=M^{*}(\omega) \omega \mathbf{v}
$$

reduces to $M^{*}$ from Eq. (4). On the contrary, if $q^{2} R_{i}^{2} \gg 1$ the associated mass $M^{*}(\omega)$ acquires a noticeable frequency dependence. In both cases oscillations of a sphere in ideal liquid involve energy dissipation through emission of sound waves.

Taking into account Eq. (5), in the presence of driving field $e E_{\|}(t)=e E_{0} \exp (-i \omega t)$ the equation set (1) is replaced by the following equations:

$$
\begin{gathered}
-m_{i} \omega^{2} x=-k\left(x-x_{1}\right)+e E_{0}, \\
-M \omega^{2} x_{1}=-k\left(x_{1}-x\right)+F_{i d}\left(\omega, v_{1}\right) \quad v_{1}=-i \omega x_{1} .
\end{gathered}
$$

Here the force $F_{i d}\left(\omega, v_{1}\right)$ from (5) contains efficient friction for the cluster motion in the solvent which accounts for the frequency dependent associated cluster mass $M=4 \pi R_{i}^{3} \rho_{s} / 3, \rho_{s}>\rho_{l}$ being the effective density in the neutral part of the cluster.

For massive clusters (strongly asymmetric normal electrolytes) the typical frequency range can shift to lower values compared to Eq. (5) with $\delta(\omega) \simeq R_{i}$, where $\delta(\omega)$ is the viscous penetration depth. If the external part of the problem is again modelled with a sphere of radius $R_{S}$ (which does not necessarily coincide with $R_{i}$ ) the Walden $[12,13]$ rule is assumed to be valid for considered clusters, i.e.,

$$
\sigma \eta=\mathrm{const}
$$

where $\sigma$ is the conductivity (or, equivalently, mobility), $\eta$ is the solvent viscosity, then the solvent response to the 
motion of sphere is described by the Stokes force $F_{S}(\omega, v)$ with the Fourier components

$$
\begin{aligned}
& F_{S}(\omega, v)=6 \pi \eta R_{c}\left(1+\frac{R_{S}}{\delta(\omega)}\right) v(\omega)+ \\
& +3 \pi R_{s}^{2} \sqrt{\frac{2 \eta \rho}{\omega}}\left(1+\frac{2 R_{S}}{9 \delta(\omega)}\right) i \omega v(\omega),
\end{aligned}
$$

where $v$ is the sphere velocity, $\delta(\omega)=\left(2 \eta / \rho_{l} \omega\right)^{1 / 2}$ is the already mentioned dynamic penetration depth, $\rho_{l}$ is the liquid density, and $\eta$ is its viscosity. Equations of motion are now written in the form of $(6),(7)$ with $F_{i d}\left(\omega, v_{1}\right)$ defined by Eq. (5) replaced with $F_{S}(\omega, v)$ (9). Details of arising dynamics were studied in Ref. 14.

2. It is natural to start the estimation of the strength of coupling between the charge and neutral shell of the cluster by considering single charged droplets. Let for definiteness the charge is assumed to be a single proton with mass $m_{p}$, interacting with adjacent $N$ water molecules each having volume $V_{\mathrm{aq}}$ and effective mass $m_{\mathrm{aq}}$. The corresponding effective parameters of the droplet are:

$$
M=N m_{\mathrm{aq}}, \quad \frac{4}{3} \pi R^{3}=N V_{\mathrm{aq}}
$$

Inside the droplet, the proton is repelled from its surface by image forces so that in equilibrium it resides at the droplet center. The energy $\delta U(r)$ associated with its displacement from the center of the sphere is

$$
\delta U(r) \simeq \frac{e^{2}(\varepsilon-1)}{\varepsilon(\varepsilon+2)} \frac{r^{2}}{R^{3}} .
$$

Accordingly, the restoring force $f_{\varepsilon}=-\partial \delta U(r) / \partial r$ contains the stiffness coefficient $k$ :

$$
f_{\varepsilon}=-k x, \quad k=\frac{e^{2}(\varepsilon-1)}{\varepsilon(\varepsilon+2)} \frac{2}{R^{3}} .
$$

Here $\varepsilon$ is the static dielectric constant of water, and $R$ is taken from Eq. (10).

Based on Eqs. (11), (11a) we shall start our estimates with finding the proton localization radius in the potential (11). In the classical treatment, one obtains the so-called Bierum radius following from the estimate

$$
\frac{\delta U\left(r_{T}\right)}{T} \simeq 1, \text { or } \frac{r_{T}^{2}}{R^{2}} \simeq \frac{\varepsilon T R}{e^{2}} .
$$

At temperatures not too different from room temperature the ratio $r_{T} / R$ for water does not exceed unity (which should be expected from the physical essence of the problem) until $R<10^{-7} \mathrm{~cm}$.

Accordingly,

$$
\omega_{p}^{2} \simeq k / m_{p}=\frac{e^{2}(\varepsilon-1)}{\varepsilon(\varepsilon+2)} \frac{2}{m_{p} R^{3}} .
$$

The scale of arising frequency $\omega_{p} \sim 3 \cdot 10^{12} \mathrm{~s}^{-1}$ is noticeably (at least by an order of magnitude) below the typical frequencies observed in experiments [7-10].

An alternative to the mechanism (10-13) is the scenario with solidified neutral shell of the cluster. Assuming the solid part to be homogeneous and the domain where force is applied to be point-like, one can estimate the coupling coefficient $k$ in the following way. Since the longitudinal sound velocity in ice is known to be $s \simeq 4000 \mathrm{~m} / \mathrm{s}$ while the lattice spacing (which is of order of the charge localization length $R_{i}$ ) is $a \simeq 3 \AA$, one has the following estimate for $k$ :

$$
k \sim\left(\frac{s}{a}\right)^{2} m_{\mathrm{aq}} \sim 5 \cdot 10^{3} \mathrm{dyn} / \mathrm{cm} .
$$

Estimation of the frequency (3) with the above value of $k$ and proton mass $m_{p} \simeq 1.6 \cdot 10^{-24} \mathrm{~g}, m_{\mathrm{aq}} \simeq 10^{-22} \mathrm{~g}$, yields

$$
\omega_{s} \simeq 6 \cdot 10^{13} s^{-1}
$$

Understanding the qualitative nature of this estimate, we note that the scale of $\omega_{s}(15)$ is comparable with the typical frequencies reported in [7-10] thus indicating correctness of physical picture of phenomenon occurring in dynamics of charged nanodroplets (elastic coupling between the central charge and the shell).

3. One of the most promising charged clusters in liquids is the cation $\mathrm{He}_{4}^{+}$(snowball) (as well as the anion (bubble)) in helium. Occupying an important place among various polaron-like structures, the snowball and bubble possess unique quantitative characteristics: at low temperatures the effective mass $M_{\mathrm{eff}}^{\text {snow }}$ is of the order of 32 helium atom masses (the bare mass being one helium atom mass), while $M_{\mathrm{eff}}^{\text {bub }}>200 m_{4}$ with the bare mass equal to that of a single electron. The effective masses $M_{\text {eff }}^{\text {snow }}$ and $M_{\text {eff }}^{\text {bub }}$ can be measured with different techniques (e.g., the cyclotron resonance, excitation of 2D plasma resonances [15] or eigenfrequencies at the surface levels [16]), and there exist interesting data on the temperature and pressure dependence of $M_{\mathrm{eff}}^{\text {snow }}(T, P)$ [17], etc. However, even in these advanced (compared with achievements in dynamics of normal electrolytes) problems the structure of $M_{\mathrm{eff}}^{ \pm}$is still unclear. Thus, estimates of $M^{+}$in the Atkins model [18] yield the value of $M^{+} \geq 50 m_{4}$ already for the core part substantially exceeding the observed mass [15]. Similar discrepancies with numerical values occur in the problem of structural resonances for bubble-quasiparticles currently providing the only example of clusters where excitation with external field was realized (internal photo-transitions for electron in the bubble) [19]. Available calculations of the frequencies of electron transitions assume the spherical potential barrier confining the electron to the bubble to be stationary (fixed). However, simple analysis presented above already indicates that some caution is needed here since for the bubble almost the entire mass has an asso- 
ciated origin. At high frequencies corresponding to electron transitions $M^{*}$ is close (as will be seen later) to zero so that the electron transition should be considered taking into account the finite value of the associated mass $m_{e}+M^{*}$ which has not been done yet. Thus the general problem on the structure of the total effective mass and the details of formation of its associated component is actually important in the case of helium ions.

For more detailed treatment we note that according to Eq. (5) the frequency dependences of both imaginary and real parts of the force $\mathbf{F}_{i d}$ are due to a finite value of the sound velocity $s_{l}$ in the ideal liquid. This velocity is less than the sound velocity in the snowball solid core $\left(s_{S} \sim 500 \mathrm{~m} / \mathrm{s}\right.$ [20]) and a fortiori exceed the typical velocities of the electron origin in the bubble. Therefore, the structural resonances for snowballs and bubbles (the resonances given by Eq. (3)) should be expected in the frequency range well above frequencies where the associated mass dispersion occurs. Adopting the dimensionless variables where frequencies and velocities are normalized to $\omega_{0}=s_{l} / R_{i}$ and $v_{0}=e E /\left(M^{*} \omega_{0}\right)$, respectively, the equation set (6), (7) can be reduced to a single equation for $v_{1}=-i \omega x_{1}$ which takes the form

$$
v_{1}=-\frac{1}{\omega\left[i \zeta(\omega)+2\left(1-\omega^{2} / \Omega^{2}\right) f(\omega)\right]},
$$

where

$$
\begin{gathered}
\zeta(\omega)=\frac{m_{i}+M\left(1-\omega^{2} / \Omega^{2}\right)}{M^{*}}, \quad \Omega=\frac{\sqrt{k / m_{i}}}{\omega_{0}}=\frac{s_{s}}{s_{l}} \frac{R_{i}}{a} \\
f(\omega)=\frac{-\omega^{3}+i\left(2+\omega^{2}\right)}{4+\omega^{4}} .
\end{gathered}
$$

In the limit $\Omega / \omega_{0} \rightarrow \infty$, i.e., $k \rightarrow \infty$, Eq. (17) yields for the real and imaginary parts of the velocity

$$
\begin{gathered}
\operatorname{Re} v_{1}=\frac{4+\omega^{4}}{\omega} \frac{\omega^{3}}{\omega^{6}+\left[2+\omega^{2}+\mu\left(4+\omega^{4}\right)\right]^{2}} ; \\
\operatorname{Im} v_{1}=\frac{4+\omega^{4}}{\omega} \frac{2+\omega^{2}+\mu\left(4+\omega^{4}\right)}{\omega^{6}+\left[2+\omega^{2}+\mu\left(4+\omega^{4}\right)\right]^{2}}, \\
\mu=\left(m_{i}+M\right) / M^{*} .
\end{gathered}
$$

Frequency dependences of $\operatorname{Re} v_{1}$ and $\operatorname{Im} v_{1}$ for different values of the parameter $\mu=\left(m_{i}+M\right) / M^{*}$ are shown in Figs. 1 and 2. Variations of these parameter allow one to get an idea on the role of the bare mass $m_{i}$ in the considered problem. In particular, the values $m_{i}=m_{e} \ll M^{*}$, $M=0$ model the limit of zero bare mass suitable for single electron bubbles (a consistent approach to this problem requires solution of the wave equation the electron). For comparison, the insets show the "usual" frequency plots for the same quantities derived in the standard Drude model (see details in Ref. 14). Qualitative difference in their

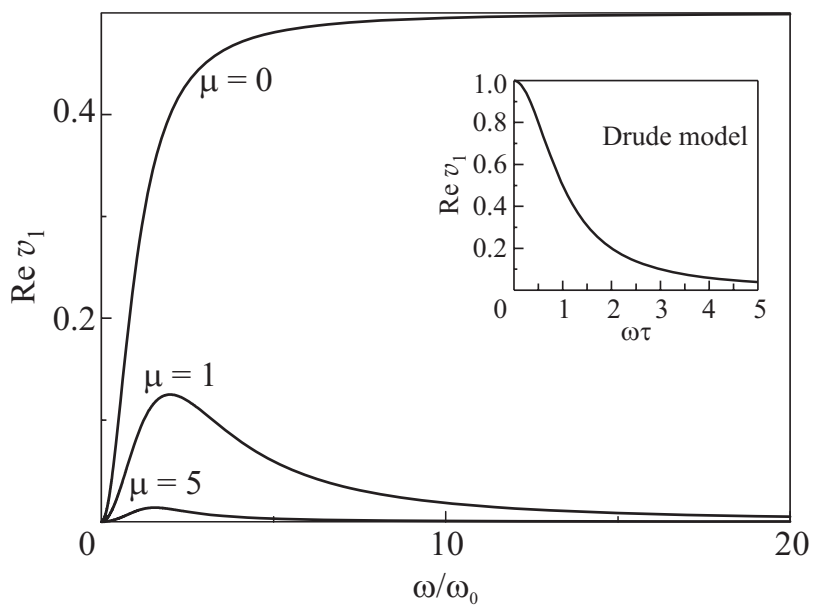

Fig. 1. $\operatorname{Re} v_{1}(\omega)$ as a function of $\omega$ for different values of the parameter $\mu$. Inset shows the usual behavior of $\operatorname{Re} v_{1}(\omega)$ for the Drude model ( $\tau$ is the momentum relaxation time).

behavior for the Drude and sound-related scenarios of the impedance.

To get a more thorough understanding of the impedance-type information presented in Figs. 1 and 2, it is useful to trace the frequency dependence of the associated mass defined by Eq. (5a). The curve (1) in Fig. 3 reveals rapid reduction of $M^{*}$ in the frequency range exceeding the critical value determined by the sound velocity in liquid and the sphere radius. Qualitatively, this critical value coincides with the position of a peak in the $M^{*}(\omega)$ curve whose existence just follows from the definition of $\mathbf{F}_{i d}$ (5) and could hardly be given any physical interpretation.

Turning back to Eq. (16), it is easily seen that the main qualitative conclusions concerning the effect of $\mathbf{F}_{i d}$ on the snowball structure resonance are related to the practical possibility (following from Fig. 3) of neglecting the imaginary part of this force (the associated mass $M^{*}$ does not have enough time to be formed) and appearance of finite damping due to emission of sound waves by the oscillating

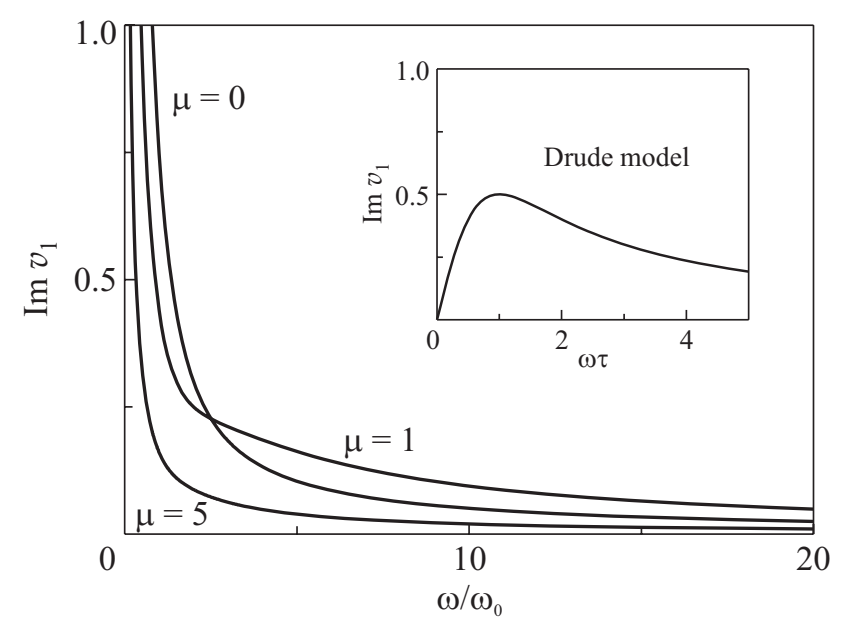

Fig. 2. $\operatorname{Im} v_{1}(\omega)$ as a function of $\omega$ for different values of the parameter $\mu$. Inset shows $\operatorname{Im} v_{1}(\omega)$ for the Drude model $(\tau$ is the momentum relaxation time). 


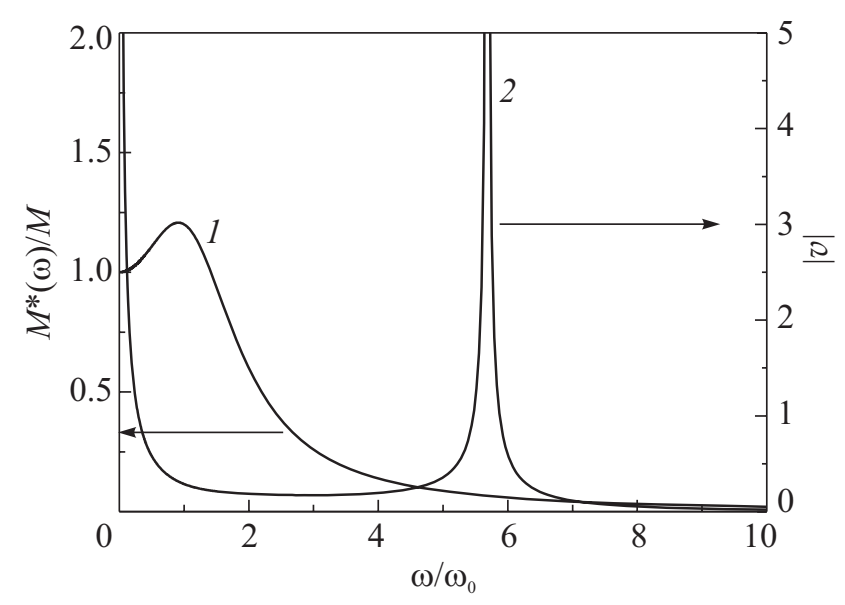

Fig. 3. Frequency dependence of the effective associated mass normalized to $M$ (curve 1, left scale) and the cluster velocity modulus for the cluster mass $M=32 m_{4}$ (curve 2, right scale).

sphere. In other words, the resonance position is actually determined by Eqs. (3) and (4). At the quantitative level this information gathered together in Fig. 3 is presented in the following way. First of all, plotted is the frequency dependence of the cluster velocity absolute value for $M=$ $=32 m_{4}$ [15]. The dimensionless frequency $\Omega$ from (17) for helium takes into account difference in the sound velocities in solid and liquid phases (the former is 2-2.5 times larger than the latter [20]) as well as the cluster size (Atkins radius) which is approximately twice as large as the interatomic distance. For convenience, shown in Fig. 3 is also the frequency dispersion of the associated mass $M^{*}(\omega)$ (curve 1 ).

To comment on Fig. 3, we note that the structure resonance for the cation in liquid helium is rather pronounced (its broadening due to emission of sound waves is not too large). In addition, it is clearly seen that the frequency dispersion of $M^{*}(\omega)$ should be taken into account. Apart from that, in the vicinity of the resonance $M^{*}(\omega) \rightarrow 0$. Bearing in mind the above results, one can hope that the actual position of the resonance yields interesting information on the elastic coupling coefficient $k$ between the core charge and the cluster shell.

Summary. A resonance approach to the study of structure of charged clusters in various electrolytes is proposed. The indicated resonances arise when the core charge and the cluster neutral shell move relative to each other and contain interesting information on the strength of elastic coupling between them. Within the corresponding formal- ism the problem of formation of "ideal" associated mass of an oscillating sphere is discussed which is directly related to the structure resonances behavior.

The work was supported by the RFBR grant No 09-0200894a and Program of the Presidium of RAS "Quantum Physics of Condensed Matter".

1. E.B. Conway, Physical Chemistry: An Advanced Treatise, H. Eyrung, D. Henderson, and W. Yost (eds.), Academic, New York (1970), Vol. IX A.

2. H.L. Friedman and C.V. Krishnan, Water: A Comprehensive Treatise, F. Franks (ed.), Plenum, New York, (1973), Vol. 3.

3. N.H. March and M.P. Tosi, Atomic Dynamics in Liquids, Macmillan, London (1976).

4. J.-P. Hansen and I.R. McDonald, Theory of Simple Liquids, Elsevier (2006).

5. P. Debye, J. Chem. Phys. 1, 13 (1933).

6. R. Zana and E. Yeager, J. Phys. Chem. 71, 521 (1967).

7. M. Miyazaki, A. Fujii, T. Ebata, and N. Mikami, Science 304, 1134 (2004).

8. J.-W. Shin, N.I. Hammer, E.G. Diken, M.A. Johnson, R.S. Walters, T.D. Jaeger, M.A. Duncan, R.A. Christie, and K.D. Jordan, Science 304, 1137 (2004).

9. Jeffrey M. Headrick, Eric G. Diken, Richard S. Walters, Nathan I. Hammer, Richard A. Christie, Jun Cui, Evgeniy M. Myshakin, Michael A. Duncan, Mark A. Johnson, and Kenneth D. Jordan, Science 308, 1765 (2005).

10. L.I. Yeh, M. Okomura, and J.D. Myers, J. Chem. Phys. 91, 7319 (1989).

11. L.D. Landau and E.M. Lifshitz, Hydrodynamics, Nauka, Moscow (1986) (in Russian).

12. P. Walden, Z. Electrochem. Angew. Phys. Chem. 12, 77 (1906).

13. P. Walden, Z. Phys. Chem. 55, 207 (1906).

14. I. Chikina, S. Nazin, and V. Shikin, Europhys. Lett. 80, 26003 (2007); I. Chikina, S. Nazin, and V. Shikin, ibid. 85, 16001 (2009).

15. Mary L. Ott-Rowland, Vince Kotsubo, Joe Theobald, and Gary A. Williams, Phys. Rev. Lett. 49, 1708 (1982); C. Mellor, C. Muirhead, J. Travers, and W. Vinen, J. Phys. C21, 325 (1988).

16. J. Poitrenaud and F.I.B. Williams, Phys. Rev. Lett. 29, 1230 (1972).

17. A. Dahm and T. Sanders, J. Low Temp. Phys. 2, 199 (1970).

18. K. Atkins, Phys. Rev. 116, 1339 (1959).

19. C.C. Grimes and G. Adams, Phys. Rev. B41, 6366 (1990); Phys. Rev. B45, 2305 (1992).

20. R. Wanner and J.P. Franck, Phys. Rev. Lett. 24, 365 (1970). 